\title{
A Case of Multiple Gastrointestinal Obstructions in a Newborn Presenting With Pseudo-Bartter's Syndrome
}

\author{
Zeynep Alp Unkar ${ }^{\mathrm{a}, \mathrm{e}}$, Nazli Kuter ${ }^{\mathrm{b}}$, Kivilcim Cerit ${ }^{\mathrm{c}}$, Asli Memisoglu ${ }^{\mathrm{a}}$, Harika Alpay ${ }^{\mathrm{d}}$, Hulya Bilgen ${ }^{\mathrm{a}}$, \\ Eren Ozek ${ }^{\mathrm{a}}$
}

\begin{abstract}
Hypochloremic metabolic alkalosis occurs in pseudo-Bartter's syndrome (PBS) without a primary renal tubular defect. Usually seen in cystic fibrosis, this syndrome can also be caused by recurrent vomiting for various reasons. We present a case of multiple gastrointestinal anomalies, each by itself capable of causing the clinical condition, in a 10-day-old infant with PBS.
\end{abstract}

Keywords: Pseudo-Bartter's syndrome; Newborn: Gastrointestinal obstruction

\section{Introduction}

Pseudo-Bartter's syndrome (PBS) is an umbrella term for clinical entities presenting with metabolic alkalosis paired with electrolyte depletion, namely hypokalemia, hypochloremia and hyponatremia, without a primary renal pathology as opposed to Bartter's syndrome in which the main abnormality is a genetically derived renal tubular defect. The most common conditions causing such a biochemical profile are cystic fibrosis, diuretic and laxative abuse, cyclic vomiting or blumia, chloride-deficient diet and congenital chloride diarrhea [1]. Urine electrolyte analysis is an easy way to distinguish between true Bartter's syndrome and PBS, excluding diuretic abuse in which chloride loss is also renal without an organic

Manuscript accepted for publication November 25, 2014

${ }^{a}$ Neonatology, Department of Pediatrics, Marmara University Faculty of Medicine, Istanbul, Turkey

bDepartment of Pediatrics, Marmara University Faculty of Medicine, Istanbul, Turkey

'Department of Pediatric Surgery, Marmara University Faculty of Medicine, Istanbul, Turkey

dPediatric Nephrology, Department of Pediatrics, Marmara University Faculty of Medicine, Istanbul, Turkey

${ }^{e}$ Corresponding Author: Zeynep Alp Unkar, Marmara Universitesi Pendik Egitim ve Arastirma Hastanesi, Fevzi Cakmak Mh. Mimar Sinan Cd. No. 41 Pendik 34899, Istanbul, Turkey. Email: md.zeynepalp@gmail.com

doi: http://dx.doi.org/10.14740/ijcp174w defect $[2,3]$.

Other than cystic fibrosis, vomiting - disregarding the reasons - seems to be the most common reason for PBS. PBS caused by gastrointestinal (GI) losses due to obstruction and vomiting reported in literature may or may not be associated with other co-existing anomalies $[4,5]$. There are rare neonatal cases with PBS, in the early postnatal period, related to their mothers' eating disorders and hyperemesis gravidarum, and the mothers also have laboratory findings similar to their babies $[6,7]$.

Pseudo-Bartter's syndrome in neonates due to GI obstruction is a rare condition. The case presented in this report is an incidental one, having been brought to the emergency department for the sole reasons of jaundice realized by the parents, and dehydration. The patient's main clinical condition was revealed after detailed history-taking and workup for hyperbilirubinemia. In infants where vomiting causes PBS, there usually is a single obstruction per case, whereas in this neonate, there are three GI obstructions, hence the reason to present the case.

\section{Case Report}

A 10-day-old female baby was admitted to the emergency department with a chief complaint of jaundice. She was born at 39th gestational week by spontaneous vaginal delivery to a gravida 2 para 128 -year-old mother. There was no history of consanguinity. The baby was discharged from the hospital on the second day of life and had no trouble with feeding or defecation for the first 2 days after discharge. However, nonbilious vomiting started on the fourth day of life and continued throughout the week prior to presentation. Shortly after each feeding (10 - 12 times/day) the baby vomited, amount close to whole meal, sometimes in a projectile fashion. The parents noticed the jaundiced sclera, and when they started having trouble waking her up for breastfeeding, they brought her to the emergency department. Upon admission to neonatal intensive care unit (NICU), physical examination revealed that the infant was severely dehydrated, with $10 \%$ weight loss and significant jaundice. She was awake and agitated, and her reflexes were normal. The blood tests showed a total bilirubin level of $21 \mathrm{mg} / \mathrm{dL}$, and her blood gas analysis was as follows: $\mathrm{pH}$ : $7.53, \mathrm{pCO}_{2}: 57.7 \mathrm{~mm} \mathrm{Hg}, \mathrm{HCO}_{3}: 47.5 \mathrm{mmol} / \mathrm{L}$, base excess: 


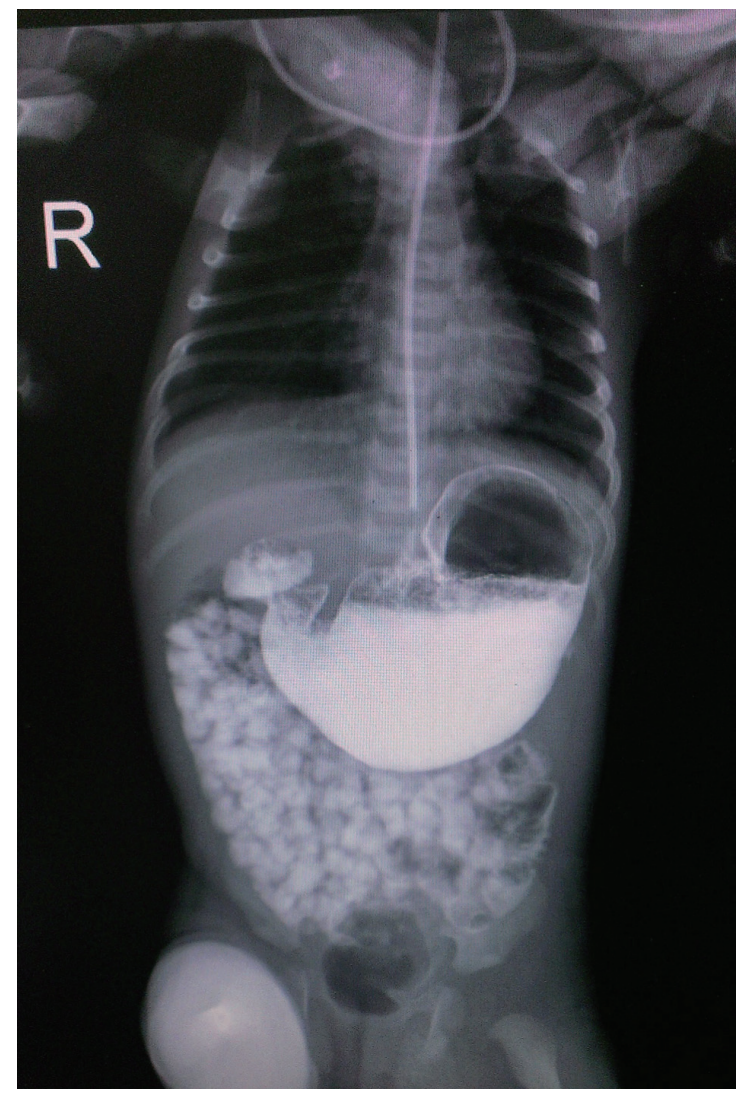

Figure 1. X-ray taken with enteral radiopaque contrast agent, revealing the enlarged stomach.

$19 \mathrm{mmol} / \mathrm{L}$ and lactate: $4 \mathrm{mmol} / \mathrm{L}$.

In the NICU, intensive phototherapy was started for unconjugated hyperbilirubinemia. After blood, urine and cerebrospinal fluid cultures were obtained, and empiric antibiotic treatment was initiated. Further blood tests revealed severe electrolyte imbalance. Plasma sodium level was $128 \mathrm{mEq} / \mathrm{L}$, potassium $3.9 \mathrm{mEq} / \mathrm{L}$, chloride $66 \mathrm{mEq} / \mathrm{L}$, calcium $11 \mathrm{mg} / \mathrm{dL}$ and phosphorous $12.4 \mathrm{mg} / \mathrm{dL}$. Therefore, the patient had hyponatremic, hypochloremic and hypokalemic metabolic alkalosis. The spot urine electrolyte levels were normal. The initial diagnosis was PBS, and the fluid therapy was managed according to the electrolyte levels.

Due to the history of persistent vomiting, X-rays and abdominal ultrasonography were done, and pediatric surgery was consulted to rule out intestinal obstruction and pyloric stenosis. The initial imaging was negative for both. Enteral feeding was stopped for the first days of treatment, and vomiting stopped with the cessation of feeding. On the second day of admission bilious gastric residues (maximum $30 \mathrm{~mL}$ ) started. Further investigations were done including the metabolic disease screening tests (plasma amino acid analysis, urine organic acid analysis and tandem mass spectroscopy) which were inconclusive. Acute phase reactants were negative from the beginning, and blood and urine cultures also ended up negative.

With fluid therapy metabolic alkalosis and electrolyte imbalance were corrected gradually; however, even without feed- ing, gastric residues persisted and did not decrease in amount. The empiric antibiotic treatment was completed to 7 days with the negative culture results. Enteral feeding could not be re-initiated and the pediatric surgery along with pediatric gastroenterology was consulted again. X-rays were taken with enteral radiopaque contrast agent, and the passage of the material to the colon was documented together with an enlarged stomach (Fig. 1). However, in order to rule out partial obstruction and malrotation, upper GI contrast study was done which revealed delayed passage through duodenum and a dilated proximal duodenum indicating that duodenal partial obstruction was a possibility.

The patient was transferred to the pediatric surgery ICU and underwent an exploratory surgery the next day. The operation revealed presence of distended and hypertrophic pylorus, duodenal web with pin-point opening, annular pancreas and intestinal malrotation, all of which were corrected surgically. After the operation the baby was clinically stabilized, and enteral feeding was initiated, gradually increasing in amount. When all parenteral nutrition and post-operative antibiotic treatment were stopped, the patient was discharged. In the follow-ups, the family reported no trouble with feeding or vomiting after discharge. Electrolyte imbalance and metabolic alkalosis did not recur.

\section{Discussion}

Pseudo-Bartter's syndrome is a state of extrarenal chloride depletion, excluding diuretic abuse. Though most commonly seen in cystic fibrosis, other reasons include vomiting (due to organic and inorganic reasons), laxative abuse, chloride diarrhea, etc. [2].

Vanhaesebrouck et al reported in 2007 a Turkish infant with consanguineous parents having an extra ring chromosome 8 and Hirschsprung disease presenting with PBS due to vomiting [4]. Unlike that baby, our patient had no major and minor dysmorphic features to raise the suspicion of a genetic anomaly. Also there was no history of consanguinity among the parents; therefore, a genetic consultation was not considered necessary.

Koshida et al reported in 1994 a case of intestinal malrotation causing PBS [5]. The 6-year-old boy described in the case had severe chloride deficiency secondary to episodic attacks of vomiting, and he was internalized thrice before a definite diagnosis was reached. The interesting point about their case was that midgut volvulus, usually seen in the newborn period, was detected during childhood. The case presented here is a newborn, as expected, but with additional GI anomalies, any of which could have caused vomiting and PBS single-handedly. The correcting operation would have been incomplete if the surgeons had not decided to explore further after the correction of pyloric stenosis. There are also, though very rare, cases of PBS caused by vomiting due to pyloric stenosis as presented by $\mathrm{Yu}$ et al [8] and Kim et al [9], none of which was as young as our patient nor with additional GI anomalies.

Cases presented by Mathot et al [6] and Higuchi et al [7] included neonatal PBS with mothers having the same elec- 
trolyte abnormalities as their infants. All of their histories included some sort of eating disorder or vomiting and weight loss during pregnancy. The babies were born with the existing electrolyte abnormalities which resolved a few days following birth. In our case, the pregnancy was not complicated, the mother had no problems in the perinatal period, and the baby's clinical condition deteriorated not soon after birth but in the following days.

It should be noted that in our case the patient's continuous projectile vomiting was not what disturbed the parents but jaundice. This should draw attention to the importance of informing both parents thoroughly about what is normal and what is not, before discharging a newborn.

A number of anomalies may cause PBS in the neonatal period, including GI obstructions. The patient's and the family's history is just as important a milestone for the diagnosis as physical examination, laboratory and radiological workup. The pediatric surgeon's role should not be underestimated, and a thorough exploration of the GI tract should be warranted to rule out co-existing anomalies.

\section{References}

1. Amirlak I, Dawson KP. Bartter syndrome: an overview. QJM. 2000;93(4):207-215.

2. Rodriguez-Soriano J. Bartter and related syndromes: the puzzle is almost solved. Pediatr Nephrol. 1998;12(4):315-
327.

3. Mersin SS, Ramelli GP, Laux-End R, Bianchetti MG. Urinary chloride excretion distinguishes between renal and extrarenal metabolic alkalosis. Eur J Pediatr. 1995;154(12):979-982.

4. Vanhaesebrouck S, Van Laere D, Fryns JP, Theyskens C. Pseudo-Bartter syndrome due to Hirschsprung disease in a neonate with an extra ring chromosome 8. Am J Med Genet A. 2007;143A(20):2469-2472.

5. Koshida R, Sakazume S, Maruyama H, Okuda N, Ohama $\mathrm{K}$, Asano S. A case of pseudo-Bartter's syndrome due to intestinal malrotation. Acta Paediatr Jpn. 1994;36(1):107111.

6. Mathot M, Maton P, Henrion E, Francois-Adant A, Marguglio A, Gaillez S, Collard L, et al. Pseudo-Bartter syndrome in a pregnant mother and her fetus. Pediatr Nephrol. 2006;21(7):1037-1040.

7. Higuchi R, Sugimoto T, Hiramatsu C, Kumagai T, Okutani T, Yagi S, Matsuoka T, et al. Neonatal pseudo-Bartter syndrome due to maternal eating disorder. J Perinatol. 2008;28(9):646-648.

8. Yu HR, Huang SC, Hsieh CS. Infantile hypertrophic pyloric stenosis presenting as pseudo-Bartter's syndrome and seizures: report of one case. Zhonghua Min Guo Xiao Er Ke Yi Xue Hui Za Zhi. 1998;39(3):195-197.

9. Kim YH, et al. A Case of Pseudo-Bartter's Syndrome Due to Hypertrophic Pyloric Stenosis. J Korean Pediatr Soc. 2002;45(11):1430-1434. 